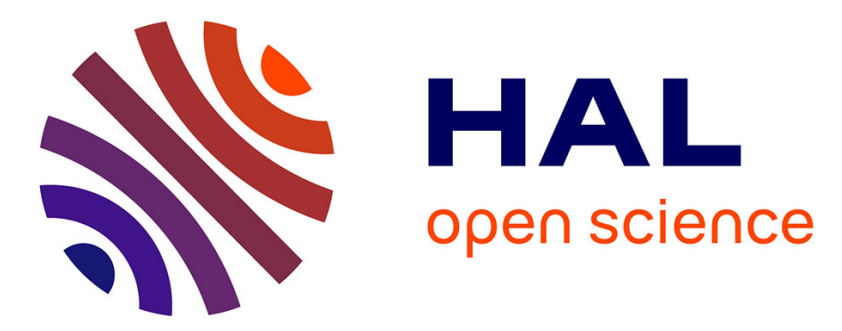

\title{
Multichannel processing for dispersion curves extraction of ultrasonic axial-transmission signals: Comparisons and case studies
}

Kailiang Xu, Dean Ta, Didier Cassereau, Bo Hu, Weiqi Wang, Pascal Laugier, Jean-Gabriel Minonzio

\section{To cite this version:}

Kailiang Xu, Dean Ta, Didier Cassereau, Bo Hu, Weiqi Wang, et al.. Multichannel processing for dispersion curves extraction of ultrasonic axial-transmission signals: Comparisons and case studies. Journal of the Acoustical Society of America, 2016, 140 (3), pp.1758 - 1770. 10.1121/1.4962491. hal-01395058

\section{HAL Id: hal-01395058 \\ https: / hal.sorbonne-universite.fr/hal-01395058}

Submitted on 10 Nov 2016

HAL is a multi-disciplinary open access archive for the deposit and dissemination of scientific research documents, whether they are published or not. The documents may come from teaching and research institutions in France or abroad, or from public or private research centers.
L'archive ouverte pluridisciplinaire HAL, est destinée au dépôt et à la diffusion de documents scientifiques de niveau recherche, publiés ou non, émanant des établissements d'enseignement et de recherche français ou étrangers, des laboratoires publics ou privés. 


\section{Multichannel Processing for Dispersion Curves Extraction of Ultrasonic Axial-Transmission Signals: Comparisons and Case Studies}
1. Kailiang Xu

Department of Electronic Engineering Fudan University, Handan Road No 220, 200433, Shanghai, China.

Sorbonne Universités, UPMC Univ Paris 06, CNRS, INSERM, Laboratoire d'Imagerie Biomédicale (LIB), 15 rue de l'école de médecine, 75006, Paris, France

\section{Dean Ta}

Department of Electronic Engineering Fudan University, Handan Road No 220, 200433, Shanghai, China.

State Key Laboratory of ASIC and System, Fudan University, Shanghai, China;

Key Laboratory of Medical Imaging Computing and Computer Assisted Intervention (MICCAI) of Shanghai, Shanghai, China.

3. Didier Cassereau

Sorbonne Universités, UPMC Univ Paris 06, CNRS, INSERM, Laboratoire d'Imagerie Biomédicale (LIB), 15 rue de l'école de médecine, 75006, Paris, France

4. $\mathrm{Bo} \mathrm{Hu}$

Department of Electronic Engineering Fudan University, Handan Road No 220, 200433, Shanghai, China.

\section{Weiqi Wang}

Department of Electronic Engineering Fudan University, Handan Road No 220, 200433, Shanghai, China.

6. Pascal Laugier

Sorbonne Universités, UPMC Univ Paris 06, CNRS, INSERM, Laboratoire d'Imagerie Biomédicale (LIB), 15 rue de l'école de médecine, 75006, Paris, France

7. Jean-Gabriel Minonzio

Sorbonne Universités, UPMC Univ Paris 06, CNRS, INSERM, Laboratoire d'Imagerie Biomédicale (LIB), 15 rue de l'école de médecine, 75006, Paris, France 
Abstract - Some pioneering studies have shown the clinical feasibility of long bones evaluation using ultrasonic guided waves. Such a strategy is typically designed to determine the dispersion information of the guided modes to infer the elastic and structural characteristics of cortical bone. However, there are still some challenges to extract multimode dispersion curves due to many practical limitations, e.g., high spectral density of modes, limited spectral resolution and poor signal-to-noise ratio (SNR). Recently, two representative signal processing methods have been proposed to improve the dispersion curves extraction. The first method is based on singular value decomposition (SVD) with advantages of multi-emitter and multi-receiver configuration for enhanced mode extraction; the second one uses linear Radon transform (LRT) with high-resolution imaging of the dispersion curves. To clarify the pros and cons, a face to face comparison was performed between the two methods. The results suggest that the LRT method is suitable to separate the guided modes at low frequency-thickness-product $(\boldsymbol{f} \cdot \boldsymbol{h})$ range; for multimode signals in broadband $\boldsymbol{f} \cdot \boldsymbol{h}$ range, the SVD-based method shows more robust performances for weak mode enhancement and noise filtering. Different methods are valuable to cover the entire $\boldsymbol{f} \cdot \boldsymbol{h}$ range for processing ultrasonic axial transmission signals measured in long cortical bones.

Keywords: Waveguides; Singular value decomposition; Linear Radon transform; Dispersion curves PACS: 43.60.Fg, 43.60.Gk, 43.80.Vj, 43.80.Jz, 43.80.Ev 


\section{INTRODUCTION}

In the past decade, significant progress has been achieved in quantitative ultrasound (QUS) assessment of cortical bone using axial transmission techniques ${ }^{1,2}$. Such techniques are intended to osteoporotic fracture discrimination ${ }^{3}$ or to fracture healing monitoring ${ }^{4,5}$. Several axial transmission techniques have been explored using either one transmitter/one receiver ${ }^{6}$, one transmitter/multiple-receiver ${ }^{7}$, or multiple-transmitter/multiple-receiver configurations ${ }^{8}$. Signal analysis developed for clinical applications are based on velocity measurement of a single signal, such as the first arriving signal (FAS) ${ }^{6}$, or a slower second energetic wave which has been interpreted as the fundamental anti-symmetrical A0 Lamb mode based on plate model ${ }^{7}$. If the cortical thickness is much smaller than the longitudinal wavelength, FAS can be seen as the S0 Lamb mode. If the cortical thickness is much larger than the longitudinal wavelength, the FAS corresponds to the non-dispersive lateral compression wave ${ }^{7}$. In between, when the thickness is comparable to the wavelength (e.g., at $1 \mathrm{MHz}$, the wavelength in cortical bone is 3 to $4 \mathrm{~mm}$ ), a physical interpretation of the FAS has yet to be provided. Besides FAS, reflection and conversion of ultrasonic body waves have been observed in the relatively thick bovine tibia ex vivo ${ }^{9}$.

Whereas the signal analysis techniques applied so far in axial transmission meet the need for simplicity and pragmatism, the corresponding biomarkers extracted from a single signal (either FAS or A0 mode) provide an incomplete biomechanical characterization of bone strength. Such a consideration has motivated the research aiming at fully characterizing the response of cortical long bones to an ultrasonic excitation. Particularly, considering that long bones actually support the propagation of multiple guided modes, several studies have shown interest in measuring and interpreting the dispersion curves of guided waves within a wideband frequency-wavenumber range ${ }^{1,2}$. Measurement of dispersion curves, along with suitable waveguide modeling, has been proposed for concurrent estimation of cortical thickness and elastic properties ${ }^{10-14}$. However, the extraction and accurate interpretation of the dispersion characteristics of guided modes propagating along the cortical shell of long bones pose difficulties, such as the high spectral density of modes, limited spectral resolution and poor signal-to-noise ratio (SNR).

Particularly, because of the limited spatial sampling or resolution achievable in clinical measurements with clinical probes (typically a few cm-long array), the traditional two-dimensional (or spatio-temporal) Fourier transform (2D-FT) ${ }^{15}$ cannot achieve a high wavenumber resolution for complete multimode separation ${ }^{2}$. From guided signal processing point of view, several methods have been described to distinguish overlapped modes and to extract the corresponding dispersion curves. These include dispersion-based short-time Fourier transform ${ }^{16}$, group velocity filtering ${ }^{17}$, time-frequency representation ${ }^{18}$, warped frequency transform ${ }^{19}$, time-frequency ridge extraction ${ }^{20,21}$, dispersion compensation ${ }^{22}$, blind identification ${ }^{13}$, generalized warblet transform ${ }^{23}$, adaptive Chirplet transform ${ }^{24}$, orthogonality relation-based method ${ }^{25}$ and compressed sensing method ${ }^{26}$. Recently, two methods taking advantage of a multiple-transmitter/multiple-receiver implementation of axial transmission have been proposed, singular vector decomposition (SVD) ${ }^{8,27}$ and linear Radon transform (LRT) ${ }^{28,29}$.

The SVD-based approach is able to significantly enhance the weak-amplitude modes by selecting the singular values and singular vectors corresponding to the signals and by filtering out the small singular values corresponding to the noise ${ }^{8}$. This method, applied in vitro on radius specimens ${ }^{14}$ and in vivo at the forearm ${ }^{10}$, has shown a good performance to estimate cortical thickness. The LRT method is widely known in seismic data processing ${ }^{30,31}$. Taking advantage of the sparse inversion, the LRT methods are capable of achieving an energy focusing or so-called high-resolution dispersive energy imaging of the wave-packets whose arrival times are linearly dependent on the propagation path ${ }^{32,33}$. The LRT methods have already been applied to study the surface-wave data for Rayleigh wave dispersive energy imaging ${ }^{34}$. Recently, the high-resolution LRT method has been introduced to analyze the ultrasonic guided signals in long bone, and 
was observed to provide enhanced resolution of the extracted dispersion curves in comparison to the 2D-FT method ${ }^{28,29}$.

While results on bone have been reported with SVD-based and LRT approach by different groups, the pros and cons as well as the applicability conditions of the two different signal processing techniques have not been thoroughly discussed. In this study, a face to face comparison is performed using SVD-based, LRT and the classical 2D-FT method. Synthetic and experimental signals on bone-mimicking phantoms and on ex-vivo human radius are analyzed.

\section{BRIEF REVIEW OF THE PROPOSED SIGNAL PROCESSING METHODS}

Typically, the frequencies used for the axial transmission measurement of long cortical bone are in the range between $50 \mathrm{kHz}$ and $2 \mathrm{MHz}$ and the typical cortical thickness of human radius and tibia are in the range between 1 to $6 \mathrm{~mm}$ approximately. According to the characteristics of the guided signals observed in different ranges of the frequency-thickness product $(f \cdot h)$, three representatives cases can be distinguished, as shown in Table 1: (1) low frequency-thickness product $f \cdot h<1 \mathrm{MHz} \cdot \mathrm{mm}$, corresponding to thin cortical bone about $1 \mathrm{~mm}$ to $4 \mathrm{~mm}$ (mainly for radius), where mainly two fundamental Lamb modes S0 and A0 are observed, (2) intermediate $f \cdot h$ range $(1<f \cdot h<6 \mathrm{MHz} \cdot \mathrm{mm})$ where multiple guided modes overlap without temporally separated wave-packets, and (3) high $f \cdot h>6 \mathrm{MHz} \cdot \mathrm{mm}$, corresponding to the thick cortical bone, e.g., tibia, which is mainly in the range of body waves propagation.

Various signal processing techniques have been reported to identify and disentangle the overlapping modes for short axial propagation distances. Table II lists the different signal processing approaches proposed for axial transmission, together with a brief description of the methods and conditions of application.

\section{THEORY AND METHODS}

\section{A. Guided Waves Dispersion}

Lamb modes in plates are classified as symmetric (S) and antisymmetric (A) modes, briefly named as Sn and $A n$ modes $(n=0,1,2, \ldots)$, which are solutions of the Rayleigh-Lamb equations ${ }^{35,36}$. The dispersion curves can be expressed as wavenumber $k$ versus $f$ or $f \cdot h$.

The 2D-FT provides a general relationship between the distance-time space $(x, t)$ and frequency-wavenumber space $(f, k)$.

$$
S(k, f)=\iint_{-\infty}^{+\infty} g(x, t) e^{j(k x-2 \pi f t)} d x d t
$$

where $g(x, t)$ is the temporal signals recorded at a series of space positions $x$. The dispersion curves can thus be obtained by locating the wavenumbers at each frequency where the amplitude of 2D-FT result $S(k, f)$ reaches the maxima.

With a given dispersion curve and an excitation signal, spectrum of the dispersive signal at distance $x_{0}$ can be computed by multiplying a phase-spectrum adjustment term $e^{-j k(f) x_{0}}$ to the spectrum of excitation. The temporal waveforms can thus be obtained by using inverse Fourier transform to the phase-adjusted spectrum of excitation. Such a procedure provides us an efficient way to synthesize the temporal signal $g(x, t)^{22}$.

\section{B. Singular Value Decomposition-based Wavenumber Extraction}

The 3D multi-emitter and multi-receiver signals matrix is denoted as $M(E, x, t)$, where $x$ is the distance sampled by $N_{r}$ receivers, and $E$ denotes different emitters, $E=1,2, \ldots, N_{e} . \mathcal{M}(E, x, f)$ is the spectrum of $M(E, x, t)$ in frequency domain. After the SVD decomposition of the $2 \mathrm{D}$ response matrix $\mathcal{M}\left(E, x, f=f_{0}\right)$ 
at each frequency $f_{0}$, we obtain two unitary matrices, i.e., an $N_{e} \times N_{e}$ matrix $U$ and an $N_{r} \times N_{r}$ matrix $V$, and an $N_{e} \times N_{r}$ rectangular matrix $\sum$ with the singular values as the diagonal entries $\sigma_{i}\left(i=1, \ldots, N_{e}\right)$. The $N_{r}$ columns of $V$, i.e., $v_{i}\left(i=1, \ldots, N_{r}\right)$, are the reception singular vectors. At a particular frequency, only the first $N_{r k}$ singular vectors $v_{i}$ associated with the most energetic singular values $\sigma_{i}\left(i=1, \ldots, N_{r k}\right)$ are retained as a basis of the received signal subspace. The wavenumber determination is then achieved by projecting a testing vector onto this basis. 27

The testing vector is expressed as an attenuated spatial plane wave with a complex wavenumber $k+j \alpha$

$$
v^{\text {test }}(k, \alpha)=\frac{1}{\sqrt{\frac{N_{r}}{2 \alpha L}\left(1-e^{-2 \alpha L}\right)}} e^{-j k x-\alpha x}
$$

Denominator term $\sqrt{\frac{N_{r}}{2 \alpha L}\left(1-e^{-2 \alpha L}\right)}$ is used to normalize $v^{\text {test }}(k, \alpha)^{27} . \alpha$ is the attenuation coefficient and in the study, a constant was adopted, i.e., $\alpha=0.05 \mathrm{~Np} \cdot \mathrm{mm}^{-1}$. Such a value is close to the average attenuation coefficients of the low-order guided modes in the bone mimicking materials ${ }^{27}$. With a constant attenuation coefficient, $v^{\text {test }}(k, \alpha)$ is a function of $k$.

At each frequency $\left(k_{0}, f_{0}\right)$, the projection of $v^{\text {test }}$ onto the $N_{r k}$ first reception singular vector basis $v_{i}\left(i=1, \ldots, N_{r k}\right)$ yields the so-called Norm function $S_{\mathrm{SVD}}(k, f)^{8}$

$$
S_{S V D}\left(k_{0}, f_{0}\right)=\sum_{i=1}^{N_{r k}\left(f_{0}\right)}\left|\left\langle v^{\text {test }}\left(k_{0}, \alpha\right) \mid v_{i}\left(f_{0}\right)\right\rangle\right|^{2}
$$

where $\left\langle v^{\text {test }}\left(k_{0}, \alpha\right) \mid v_{i}\left(f_{0}\right)\right\rangle$ is the Hermitian scalar product between the $v^{\text {test }}\left(k_{0}, \alpha\right)$ and $v_{i}\left(f_{0}\right)$. Thus, at each frequency $f_{0}$, the Norm function is a function of $k$ and the maxima correspond to wavenumbers of the guided waves presented in the received signals.

Due to the normalized characteristics of the orthogonal basis, the values of Norm function range from 0 to 1 . This value can be interpreted as follows: if a guided mode exists in the signal, the corresponding Norm function value is close to 1 ; otherwise, the value is close to $0^{8}$. Details of the SVD-based method and corresponding examples can be learned from ${ }^{8,37}$. Note that the method can be adapted to achieve a more accurate dispersion extraction when the cortical thickness is not uniform but with linear changes in the direction of wave propagation ${ }^{38}$.

\section{Linear Radon Transformation based Wavenumber Extraction}

For convenience of the inversion problem, the LRT is usually formulated as the forward relationship between the $(x, t)$ and the Radon domain. Let $g(x, t)$ represent a distance-time matrix measured by single emitter and $N_{r}$ receivers. The LRT can be defined using the following equation ${ }^{30,31}$

$$
g(x, t)=\int_{p} w(p, \tau=t-p x) d p
$$

where $\tau$ and $p$ denote the intercept time and slope parameter or phase slowness, respectively; $w(p, \tau=$ $t-p x)$ designates the signal in the $(\tau, p)$ domain. The LRT is commonly named as $\tau$ - $p$ transform or slant stack.

Equation (3) can be rewritten in frequency domain as 28,31 


$$
G(x, f)=\int_{p} W(p, f) e^{-j 2 \pi f p x} d p
$$

Using matrix notation, we have

$$
G=L W
$$

The $N_{r} \times N_{p}$ linear operator $L$ is

$$
L=\left[\begin{array}{ccc}
e^{-j 2 \pi f p_{1} x_{1}} & \ldots & e^{-j 2 \pi f p_{N_{p}} x_{1}} \\
\vdots & \ddots & \vdots \\
e^{-j 2 \pi f p_{1} x_{N_{r}}} & \ldots & e^{-j 2 \pi f p_{N_{p}} x_{N_{r}}}
\end{array}\right] \quad p=p_{1}, p_{2}, \ldots, p_{N_{p}}
$$

where $N_{p}$ is the number of the slowness sampling points.

A penalized least-squares (LS) solution to Eq. (5) has been introduced by minimizing the following cost function ${ }^{31,32}$

$$
J=\|G-L W\|_{2}^{2}+\mu Q(W)
$$

where the Lagrange multiplier $\mu$, also referred as regularization hyperpameter ${ }^{39}$, determines the trade-off between the misfit term $\|G-L W\|_{2}^{2}$ describing the data fidelity and the penalty term $Q(W)$ in Radon domain.

A typical implementation of the penalty term is to use the quadratic $l_{2}$-norm, i.e., $Q(W)=\|W\|_{2}^{2}$. So that, the LS solution can be analytically obtained as

$$
\widetilde{W}=\left(L^{H} L+\mu I\right)^{-1} L^{H} G
$$

where $L^{H}$ is the complex-conjugate transpose of $L$. It should be noticed that $L^{H} L$ is not a unitary matrix. The $W(p, f)$ in the Radon field can be readily mapped to the $(k, f)$ domain via $k=2 \pi f p^{28}$. The dispersion curves can also be obtained from $\left|S_{L R T}(k, f)\right|^{2}$ by using LRT method.

If certain non-quadratic terms, which enable to quantify the amount of sparsity of a vector, are adopted as the penalty terms, then we can focus the signal energy on the "best" subspace of the solution spaces. That is actually the sparse solution leading to the high-resolution LRT method. Therefore, the penalty term actually controls the high resolution constraints and also indicates the sparsity of the results. Two typical non-quadratic penalty terms, i.e., $l_{1}$-norm $Q(W)=\|W\|_{1}$ and Cauchy norm, are usually adopted for achieving sparsity. The Cauchy norm penalty term is defined as ${ }^{33}$

$$
Q(W)=\sum_{i=1}^{N_{p}} \ln \left[1+W\left(p_{i}, f\right) / \varepsilon^{2}\right]
$$

where the scale factor of the Cauchy distribution $\varepsilon^{2}$ actually indicates the default power in absence of hyperbolic events ${ }^{33}$. According to the discrepancy principle, a proper $\varepsilon^{2}$ value should be selected to ensure that the misfit matches the power of noise ${ }^{40}$. The one-dimension Brent parabolic interpolation method has been used to compute the epsilon ${ }^{40,41}$. It is fixed as a constant $\varepsilon=0.8$ in $^{29}$. In the study, $\varepsilon=1$ was used for the Cauchy LRT computation. Although there is no analytical solution to high-resolution LRT, it can be solved efficiently by conjugate gradient technique. Details of that can be found in literature ${ }^{39,40}$.

The trade-off curve, so-called L-curve ${ }^{42}$, is usually applied for empirical optimization of the $\mu$ value for the penalized LS-LRT and high-resolution LRT method. A small hyperparameter $\mu$ value leads to a solution with minimized misfit term but with less energy focusing; conversely, a larger $\mu$ value can achieve a high resolution by emphasizing the regularization term. For simplicity and methodology comparison, a 
fixed value of trade-off $\mu=0.05$ were used for the results presented in Section IV. Detailed discussion of trade-off parameter using L-curve can be found in Section V(1).

\section{Experiments}

Experiments are achieved using an array transducer consisting in 5 emitters and 24 receivers (Vermon, Tours, France) associated with a specific driving electronic device (Althaïs, Tours, France). The pitch of the array transducer is $0.8 \mathrm{~mm}$ and the length and width of each rectangular element are $0.8 \mathrm{~mm}$ and $8 \mathrm{~mm}$, respectively. The central frequency is $1 \mathrm{MHz}$ and the $-6 \mathrm{~dB}$ bandwidth goes from 0.5 to $1.6 \mathrm{MHz}^{10}$.

For case II in Table I, experiments are carried out in two phantoms, i.e., a 4 mm-thick bone-mimicking material (Sawbones, Pacific Research Laboratory Inc, Vashon, WA) and $2.5 \mathrm{~mm}$-thick ex-vivo human radius. A $25.5 \mathrm{~mm}$-thick polymethylacrylate (PMMA) plate is also measured for case III in Table 1. Ultrasound gel (Aquasonic, Parker Labs, Inc, Fairfield, NJ) is used to ensure the coupling between the probe and the sample. The characteristics of the specimens are listed in table III. $V_{T}$ is the shear velocity, and $V_{L \|}$, and $V_{L \perp}$ are the pure compression bulk wave velocities in the direction parallel and normal to the direction of the fibers (bone-mimicking material) or to the long axis of the bone (human radius). The density and thickness are denoted by $\rho$ and $h$. Typical values for human radius density, shear and longitudinal velocity, derived from the literature, are used for computation of the theoretical dispersion curves of the $2 \mathrm{D}$ transverse isotropic free plate model ${ }^{14}$. The average thickness of the human radius specimen was obtained by X-ray computed tomography (XtremCT, Scanco Medical, Bruttisellen, Switzerland).

\section{RESULtS}

\section{A. Synthetic signals, narrow wavenumber-band Lamb modes SO and AO}

This example corresponds to case I in Table I. Two fundamental narrow $k$-band $(0<k \leq 2 \mathrm{rad}$. $\mathrm{mm}^{-1}$ ) Lamb modes $\mathrm{A} 0$ and S0 on a $2 \mathrm{~mm}$-thick bone-mimicking plate were synthesized with peak-to-peak amplitudes of 1 and 0.3, respectively (see Fig. 1a). A Gaussian random noise was added with SNR of $30 \mathrm{~dB}$. The SNR is defined as the ratio of the power of the signal and that of the noise. Fig. 1b presents the 2D-FT results in the $(k, f)$ domain. After SVD decomposition, the singular values were normalized in $\mathrm{dB}$ scale. Those singular vectors associated with singular values above the threshold $21 \mathrm{~dB}$ were remained as the signal subspaces and the rest were filtered out as noise (see Fig. 7a ). The SVD result is depicted in Fig. 1c. Fig. $1 \mathrm{~d}$ is the $(\tau, p)$ result obtained by LRT with $l_{2}$-norm. Since there is a large velocity difference between the SO and A0 modes (see Fig. 1a), they are projected as two separate regions in the $(\tau, p)$ domain (Fig. 1d). As shown in Figs. 1e-f, the high-resolution LRT results using $l_{1}$-norm and Cauchy norm are able to significantly concentrate the $k-f$ energy of the narrow wavenumber-band and S0 and A0 modes. The colors of the $k-f$ and $\tau-p$ energy distribution present the mode energy with highest values in red and lowest values in blue.

\section{B. Phantom signals}

\section{(1) Wide wavenumber-band and multiple guided modes}

This example corresponds to case II in Table I. Figure 2 presents the experimental signals measured in a $4 \mathrm{~mm}$-thick bone-mimicking plate. Fig. $2 \mathrm{a}$ is the distance-time diagram of the array-signal. As shown by the 2D-FT and SVD $k-f$ results (Figs. $2 b-c$ ), the detectable wavenumber dispersion is in the range of $0<$ $k<4 \mathrm{rad} \cdot \mathrm{mm}^{-1}$ with more than 5 modes. The experimental SNR is around 60dB. Those singular vectors associated with singular values higher than an heuristic threshold of $20 \mathrm{~dB}$ were remained as the signal subspaces and the rest were filtered out as noise. Fig. $2 \mathrm{~d}$ plots the $l_{2}$-norm-based energy distributions in the $(\tau, p)$ domain. Figs 2e-f depict the high-resolution LRT results of the multimode energy distribution in $k-f$ field using $l_{1}$-norm and the Cauchy norm. 
Compared with the 2D-FT method, using the SVD-based method, the multimode dispersion curves can be identified with an enhancement of the weak modes, e.g., A0, A1, S0, S4 etc., and also the low-amplitude $\mathrm{S} 0$ and $\mathrm{A} 1$ mode energy in wavenumber range of $3<k<4 \mathrm{rad} \cdot \mathrm{mm}^{-1}$. In Fig. 2d, the LRT method can obtain a projection in the $(\tau, p)$ domain with focused energy points. But as shown in Figs. 2e-f, the concentrated region in the $(\tau, p)$ domain only represents the strong modal energy close to the center frequency, which actually cannot lead to an effective reconstruction of the dispersion trajectories of the weak modes. For example, the A0, A1 and S4 modes, whose energy is far from the center frequency of the probe, are not clearly depicted on the LRT results.

\section{(2) Body waves}

This example corresponds to case III in Table I. A $25.5 \mathrm{~mm}$-thick PMMA plate was measured to obtain the signals mainly consisting of ultrasonic body waves. The experimental SNR is as the previous example around $60 \mathrm{~dB}$. As ultrasonic body waves have been investigated in a $6.5 \mathrm{~mm}$-thick bovine bone ex vivo ${ }^{9}$, such a very-thick plate is also prepared to clarify the performance of the two methods in the high $f \cdot h$ range. It should be noted that $25.5 \mathrm{~mm}$-thick waveguides are unlikely to be encountered in human cortical bone whose thickness varies from less than a millimeter to a few millimeters at best.

Different from the highly dispersive guided modes in the thin plates (Table I, case II), in a thick plate, there mainly exist body waves propagating as temporally separated wave-packets. As shown in Fig. 3a, there are five different wave-fronts in the distance-time diagram with different ray paths. $\mathrm{Pd}$ and $\mathrm{Sd}$ represent the longitudinal and shear waves axially propagating along the plate surface. PrP and $\mathrm{SrS}$ correspond to the first reflection of the longitudinal and shear waves on the bottom wall. PrS corresponds to the longitudinal-to-shear wave conversion when the $\mathrm{P}$ wave is reflected on the bottom surface of the plate. The markers on Fig. 3a were computed according to the distance-velocity relationship. Figs. 3b-c are the 2D-FT and SVD $k-f$ results, respectively. After projecting the array-signal from distance-time domain to Radon field, accurate slowness and amplitude of each wave-packet can be obtained from those energy focusing maxima in $\tau-p$ domain, which is convenient for detecting the components with different ray paths. Furthermore, such a slant-stack operator is very suitable to detect the weak components (see Fig. 3d). However, as shown in Figs. 3e-f, for the signals measured in high $f \cdot h$ range, there is still no evidence that the $l_{1}$-norm and Cauchy norm LRT method can improve the $k$-f resolution for better dispersive energy imaging. The SVD-based method still provides a result with best dispersion energy extraction in $k-f$ domain.

\section{Ex vivo guided signals analysis in a human radius}

This example corresponds to case II in Table I. The guided signals measured from an ex-vivo 2.5 mm-thick human radius can be seen in Fig. 4a. Figs. 4b-c are the 2D-FT and SVD $k-f$ results, respectively. Fig. $4 d$ is the multimode energy distributions in the $(\tau, p)$ domain using the $l_{2}$-norm LRT method. The experimental SNR is around 55dB (see Fig. 7b). The threshold of singular values is $20 \mathrm{~dB}$. Figs. $4 \mathrm{e}$-f show the $k-f$ energy distribution obtained by $l_{1}$-norm and Cauchy norm high-resolution LRT methods. Comparing with the LRT and 2D-FT method, SVD-based method is capable of detecting the noise polluted A0 and $\mathrm{S} 0$ mode and also part of A1, A2, S1 and S2 modes in wideband frequency-thickness range. Similar to the $4 \mathrm{~mm}$-thick phantom signals (Fig. 2), because of high dispersive characteristics, the wideband guided modes in the human radius cannot be readily separated and enhanced in the Radon field. The resolution improvement of the fundamental A1, S1 and S2 modes can be observed close to center frequency bandwidth in Fig. $4 \mathrm{e}$ using the $l_{1}$-norm LRT method, but for the relatively weak and wideband modes, e.g., A0, A2 and S0, the LRT cannot provide sufficient mode enhancement. As shown in Fig. 4f, due to the improper value of the regularization parameter, it seems that the Cauchy norm LRT method enforces the results with "all-zero" solution. The reason of that will be discussed in Section V. 
In this study, we performed a face to face comparison between two signal processing approaches, namely the SVD-based and LRT method, which have been recently proposed to extract the dispersion curves of guided waves transmitted in long bone. To this goal, the methods were applied to synthetic signals and experimental signals recorded on a bone-mimicking plate and on a human radius ex vivo.

\section{A. Parameter optimization of the LRT and SVD-based method}

The hyperparameter $\mu$ of the LRT methods, which controls the trade-off between data fidelity and mode energy concentration (or sparseness), can be heuristically determined by the L-curve. The optimal value of hyperparameter, usually determined on the "elbow" of L-curves, actually corresponds to the maximal curvature point where the misfit and penalty terms are minimized together ${ }^{28,42}$. For the SVD-based method, a SNR threshold is used to selectively separate the noise and signal spaces.

\section{(1) Hyperparameter $\mu$ of the LRT method}

It has been observed in Section IV that for case I in Table 1, the LRT methods provide similar $k-f$ dispersion loci as 2D-FT method; but for cases II and III, the over-sparse solutions of $k$-f energy distributions are readily to be obtained when using the high-resolution LRT methods. To clarify the reason of that, the L-curves are investigated for case I: synthetic signals of narrow wavenumber-band Lamb modes $\mathrm{A} 0$ and $\mathrm{S} 0$ on a $2 \mathrm{~mm}$-thick phantom plate (Fig. 5) and for case II: $2.5 \mathrm{~mm}$-thick human bone ex vivo (Fig. 6). Strictly speaking, the hyperparameter $\mu$ needs to be optimized as a function of $f$, slowness, penalty term and misfit term. Therefore, different L-curves at different frequencies are shown at 3D space of $\mu$, penalty term and misfit term. The optimal values of $\mu$ parameter are searched from $2^{-15}$ to $2^{7}$ in different bandwidths of the interest.

As shown in Fig. 5, for the narrowband case, the L-curves are computed with the slowness range of $0<$ $p<2.56 \mu \mathrm{s} \cdot \mathrm{mm}^{-1}$ and frequency $0.1<f<0.7 \mathrm{MHz}$. A group of stable L-curves is obtained with values of the hyperparameter $\mu=0.001,0.001$ and 0.015 for $l_{2}$-norm, $l_{1}$-norm and Cauchy norm, respectively. Thus, for Case I (see Fig. 1), both high resolution and noise filtering can be achieved using LRT methods with a fixed $\mu$ value for all frequencies.

The L-curves obtained from the signals of a $2.5 \mathrm{~mm}$-thick human bone ex vivo (case II) are depicted in Fig. 6, with the slowness and frequency ranges of $0<p<2.56 \mu \mathrm{s} \cdot \mathrm{mm}^{-1}$ and $1.1<f<1.6 \mathrm{MHz}$. According to the L-curves, small hyperparameter values of $\mu<1$ are still preferable for this case. However, different from Fig. 5, even using such a small $\mu$ value, most of the penalty terms are still obtained with the values below 0.01 , which actually indicates the "all-zero" solutions. Such a result explains why in Fig. 4, mode enhancement cannot be achieved by using the LRT methods in bandwidth of $1.1<f<1.6$ $\mathrm{MHz}$.

\section{(2) SNR threshold of the SVD-based method}

The performance of the SVD-based method mainly depends on the singular value selection, which can be optimized by a SNR threshold. Fig. 7 presents the 5 singular values $\sigma_{i}$ as functions of the frequency in $\mathrm{dB}$ scale, where the signals are obtained in (a) case I: synthetic signals, narrow wavenumber-band Lamb modes A0 and S0 on a 2 mm-thick Sawbones plate (see Fig. 1), and (b) case II: 2.5 mm-thick human bone ex vivo (see Fig. 4). Our typical experimental signals are recorded with general SNR around $60 \mathrm{~dB}$, but for some low-amplitude modes, the SNR can be less than $10 \mathrm{~dB}$, for example, the $\sigma_{4}(f)$ in dash line between $0.5 \mathrm{MHz}$ and $1 \mathrm{MHz}$ (Fig. 7b). It can be found that in both cases, SNR thresholds can be heuristically determined from the $\sigma_{i}$ functions.

\section{B. Application Condition}

For guided signals measured on a relatively large reception length $(>50 \mathrm{~mm})$, the $2 \mathrm{D}$-FT method is able to characterize the dispersion curves of several fundamental Lamb modes, e.g., S0, A0 and S1 in the plate 
model, or longitudinal guided modes, e.g., $\mathrm{L}(0,1), \mathrm{L}(0,2)$, and $\mathrm{L}(0,3)$ in the cylindrical model ${ }^{43,44}$. However, the limited spatial sampling achievable with clinical probes (typically a few $\mathrm{cm}$-long transducer arrays) results in a poor wavenumber resolution with the consequence that only high-amplitude and non-overlapped modes can be readily identified using the classical 2D-FT method. Such a limitation of the 2D-FT method can be improved by using the LRT methods and SVD-based method. However, different application conditions of the two methods should be taken into account.

\section{(1) LRT methods}

The merits of the LRT methods originate from the theory of the Radon transform. The linear-path functions, i.e., wave-packets in rays, can be projected to the Radon domain as different energy foci along the linear slant-stack operator. Furthermore, the inversion based sparse technique, so-called high-resolution LRT method, can be employed to sharpen the $\tau-p$ and $k-f$ resolution. As a result, only the less dispersive modes with clear temporal rays can be concentrated as foci by using high-resolution LRT methods with sparsity.

For case I (see Fig. 1), if the wave-packets actually propagate at significantly different velocities and if the temporal overlapping is mainly caused by the short propagation distance instead of dispersion, then the modes can be perfectly separated by the slowness range selection in the Radon domain. Furthermore, considering the reversibility of the LRT method between $(k, f)$ and $(x, t)$ fields, the LRT method is capable of providing another good solution to separate some narrowband modes, for example, to extract the slowest fundamental A0 modes in long bone ${ }^{29}$. The extraction of A0 mode in $\tau-p$ field might be more efficient than the temporal wave-packets extraction using the so-called group velocity mask filtering ${ }^{45}$.

However, for case II (Figs. 2 and 4), i.e., wideband multimodal signals with high attenuation and dispersion, it has been shown that the LRT methods can only enhance the resolution of some high-amplitude modes close to the center frequency of the probe, which actually fails to achieve a wideband dispersion curves extraction.

For case III (Fig. 3), the axial transmission signals, measured from the $25.5 \mathrm{~mm}$-thick PMMA plate, mainly consist of non-dispersive body waves, which are similar to the seismic signals measured from the large-scale media with multiple ray-paths. The LRT methods are able to extract the individual temporal wave-packets propagating along different paths, even for the reflected signals with low amplitudes. The slowness of each wave-packet can be directly read from the $\tau$ - $p$ domain. The results suggest that the LRT methods are suitable for extracting FAS $^{6}$ and other multipath body waves ${ }^{9}$ in the long bone.

A beamforming and angle steering strategy at the emission stage can be used to obtain the ultrasonic guided modes with narrowband phase velocity spectrum leading to relatively clear ray contributions in the distance-time diagram ${ }^{46}$. For instance, the phase velocities of the multimode signals are approximately in a range of 3 to $5 \mu \mathrm{s} \cdot \mathrm{mm}^{-128}$. In such case, although mode dispersion and temporal overlapping exist, because of the presence of relatively clear rays for the different modes, the high-resolution LRT method can still be used to improve the resolution of $k-f$ dispersion curves imaging. However, without enough enhancement of the low-amplitude modes, the LRT methods usually provide identical maxima loci in comparison to 2D-FT. Regarding the identification of those modes with high dispersion and weak amplitude in wide $k-f$ ranges, e.g., S1 and S2 modes in Fig. 4, some improvements are still necessary.

We found that (1) the high-resolution LRT methods can enhance the $k$ - $f$ resolution of some modes with narrow velocity range, e.g., two fundamental Lamb modes (S0 and A0) in Figs.1 e-f and S0, A0 and A1 and S1 modes in Fig. 4e; (2) in contrast, for wideband highly-dispersive and low-amplitude modes, it is still challenging to concentrate $k-f$ trajectories using the LRT methods, e.g., for guided waves signals in Figs. 2 and 4 corresponding to case II. The sparse assumption of the Radon projection of the linear events in the $(x, t)$ field is well satisfied when different wave-packets propagate at constant velocities (Fig. 3 case 
III). For the multimode signals with severe dispersion, the assumption is valid, when there are still clear linear events in $(x, t)$ domain, for instance, signals with only S0 and A0 modes in Fig. 1 (case I) and some seismic data presented in ${ }^{34,47,48}$. However, for case II, both the dispersion and short propagation distances of a few centimeters are responsible for modes overlapping for wavenumbers ranging from 0 to $5 \mathrm{rad}$. $\mathrm{mm}^{-1} 10,28,29$. As a consequence, there is no clear linear events observed in the wideband multimode signals (see Figs. 2 and 4), so that the low-amplitude signals cannot be effectively enhanced by using the slant-stack operator of the LRT methods. Such a challenge causes the inefficiency of the sparse penalty term, i.e., norm of the $W(p, f)$, involved in the LRT methods (Fig. 6). It could explain the difficulties during our application of the LRT methods for extracting the wideband dispersion curves, in particular for the low-amplitude multimode signals under a poor SNR (case II Figs. 2 and 4).

\section{(2) SVD-based method}

For both narrowband (case I) and wideband (case II) guided signals, the multi-emitter and multi-receiver configuration combined with the singular vectors selection strategy of the SVD allows achieving a stable performance for noise filtering and extraction of the dispersion curves.

\section{Other Potential Approaches and Improvements}

Many classical spectra estimation methods ${ }^{49}$, such as the Burg method or the multiple signal classification method (MUSIC), can be used to achieve high-resolution wavenumber estimation. Recently, sparse methods have been introduced for dispersion curves extraction. Harley et al. ${ }^{26}$ have proposed a compressed-sensing-based sparse wavenumber method for the recovery of the dispersion curves. These authors showed that the sparse penalty regularization can be directly performed using the wavenumber penalty. Such a sparse strategy in the $(k, f)$ field may be more efficient and convenient. Currently, the sparse wavenumber extraction method proposed by Harley et al. has been verified on aluminum metal plates ${ }^{26}$. The practical challenge encountered with axial transmission in cortical bone is to efficiently enhance the weak modes under the conditions of severe broadband overlapping (more than 5 modes in some frequency band, see Fig. 4). The sparse singular value decomposition (S-SVD) technique, i.e., an improved SVD-based method by using the sparse strategy in $(k, f)$ rather than the $(\tau, p)$ domain, may significantly overcome the limitation of poor wavenumber resolution ${ }^{37,50}$. Other improvements for 3D multi-emitter and multi-receiver space-time signal processing, e.g., high-dimensional seismic data processing ${ }^{51}$, might be also helpful for guided waves dispersion analysis, but the application has not been reported in community of ultrasonic bone evaluation to date. In addition, other guided modes excitation technology, e.g., coded excitation ${ }^{52-54}$ and wideband dispersion reversal method ${ }^{55}$ etc., can also be helpful to enhance the SNR of the ultrasonic axial transmission signals in the long cortical bone.

Generally speaking, in order to interpret the relatively complex guided signals, the signal processing methods should be robust enough to allow the wideband dispersion curve extraction and the low-amplitude mode detection. By retaining the singular values above noise level, the SVD-based method significantly enhances the weak mode extraction when they are poorly detected by the 2D-FT method and LRT methods. In this sense, the SVD-based method could be more suitable for signal processing of the ultrasonic guided waves in the long bone, especially for highly dispersive wideband signals, in presence of severe attenuation and low SNR.

\section{CONCLUSION}

Different signal processing methods are necessary to cover the entire cortical bone thickness range of the human long bone. The LRT methods have the advantage of the reversibility between $(\tau, p)$ and $(x, t)$ fields showing a good ability to separate modes with large velocity difference, which is suitable for data analysis of ultrasonic guided waves at low $f \cdot h$ range $(f \cdot h<1 \mathrm{MHz} \cdot m m), e . g$, signals consisting of two fundamental modes S0 and A0 with a large velocity difference presented in the study (Fig. 1). For the highly dispersive multimode signals in a broadband $f \cdot h$ range $(0<f \cdot h<6 \mathrm{MHz} \cdot \mathrm{mm})$, which are quite 
usual in the axial transmission measurement of the human long bone (Figs. 2 and 4), the SVD-based method shows more robust performances for weak mode enhancement and noise filtering. Finally, regarding computation time, the SVD-based method can be accomplished efficiently without any iterations, but the $l_{1}$-norm and Cauchy norm LRT methods are relatively time-consuming due to the reweighting strategy at each frequency.

Future work will be to improve the resolution of the SVD method using the sparse strategy, i.e., recently proposed S-SVD method ${ }^{37,50}$, which may achieve a high-resolution extraction of the dispersion curves of ultrasonic guided waves.

\section{ACKNOWLEDGMENT}

The authors also acknowledge Dr. Maryline Talmant for her constructive comments and excellent advices to improve the paper. This work was supported by the National Natural Science Foundation of China (11304043, 11327405, 11525416), the NSFC-CNRS-PICS (11511130133) and the CNRS PICS programme $n^{\circ} 07032$. 


\section{References:}

${ }^{1}$ M. Talmant, J. Foiret and J. G. Minonzio, "Guided Waves in Cortical Bone," Bone Quantitative Ultrasound, 147-179 (2011).

2 P. Moilanen, "Ultrasonic guided waves in bone," IEEE Trans. Ultrason. Ferroelectr. Freq. Control 55, 1277-1286 (2008).

${ }^{3}$ R. Barkmann, E. Kantorovich, C. Singal, D. Hans, H. K. Genant, M. Heller, and C. C. Gluer, "A new method for quantitative ultrasound measurements at multiple skeletal sites - First Results of precision and fracture discrimination D-1837-2010 C-9752-2010," J. Clin. Densitom. 3, 1-7 (2000).

${ }^{4}$ V. C. Protopappas, M. G. Vavva, D. I. Fotiadis, and K. N. Malizos, "Ultrasonic monitoring of bone fracture healing," IEEE Trans. Ultrason. Ferroelectr. Freq. Control 55, 1243-1255 (2008).

${ }^{5}$ K. Xu, D. Ta, R. He, Y. Qin, and W. Wang, "Axial transmission method for long bone fracture evaluation by ultrasonic guided waves: Simulation, phantom and in vitro experiments," Ultrasound Med. Biol. 40, 817-827 (2014).

6 A. J. Foldes, A. Rimon, D. D. Keinan, and M. M. Popovtzer, "Quantitative ultrasound of the tibia: a novel approach for assessment of bone status," Bone 17, 363-367 (1995).

${ }^{7}$ P. H. F. Nicholson, P. Moilanen, T. Karkkainen, J. Timonen, and S. L. Cheng, "Guided ultrasonic waves in long bones: Modelling, experiment and in vivo application," Physiol. Meas. 23, 755-768 (2002).

8 J. G. Minonzio, M. Talmant and P. Laugier, "Guided wave phase velocity measurement using multi-emitter and multi-receiver arrays in the axial transmission configuration," J. Acoust. Soc. Am. 127, 2913-2919 (2010).

${ }^{9}$ L. H. Le, Y. J. Gu, Y. P. Li, and C. Zhang, "Probing long bones with ultrasonic body waves," Appl. Phys. Lett. 96(11410211), 1-3 (2010).

${ }^{10}$ Q. Vallet, N. Bochud, C. Chappard, P. Laugier, and J. Minonzio, "In vivo characterization of cortical bone using guided waves measured by axial transmission," IEEE Trans. Ultrason. Ferroelectr. Freq. Control, DOI 10.1109/TUFFC.2016.2587079 (2016).

${ }^{11}$ V. Kilappa, K. Xu, P. Moilanen, E. Heikkola, D. Ta, and J. Timonen, "Assessment of the fundamental flexural guided wave in cortical bone by an ultrasonic axial-transmission array transducer," Ultrasound Med. Biol. 39, 1223-1232 (2013).

12 A. Tatarinov, V. Egorov, N. Sarvazyan, and A. Sarvazyan, "Multi-frequency axial transmission bone ultrasonometer," Ultrasonics 54, 1162-1169 (2014).

${ }^{13}$ X. Song, D. Ta and W. Wang, "Analysis of superimposed ultrasonic guided waves in long bones by the joint approximate diagonalization of eigen-matrices algorithm," Ultrasound Med. Biol. 37, 1704-1713 (2011).

${ }^{14}$ J. Foiret, J. G. Minonzio, C. Chappard, M. Talmant, and P. Laugier, "Combined estimation of thickness and velocities using ultrasound guided waves: a pioneering study on in vitro cortical bone samples," IEEE Trans. Ultrason. Ferroelectr. Freq. Control 61, 1478-1488 (2014).

${ }^{15}$ C. H. Chapman, "A new method for computing synthetic seismograms," Geophys. J. Int. 54, 481-518 (1978).

${ }^{16}$ J. Hong, K. H. Sun and Y. Y. Kim, "Dispersion-based short-time Fourier transform applied to dispersive wave analysis," J. Acoust. Soc. Am. 117, 2949-2960 (2005).

${ }^{17}$ P. Moilanen, P. Nicholson, V. Kilappa, S. L. Cheng, and J. Timonen, "Assessment of the cortical bone thickness using ultrasonic guided waves: Modelling and in vitro study," Ultrasound Med. Biol. 33, 254-262 (2007).

${ }^{18}$ V. C. Protopappas, I. C. Kourtis, L. C. Kourtis, K. N. Malizos, C. V. Massalas, and D. I. Fotiadis, "Three-dimensional finite element modeling of guided ultrasound wave propagation in intact and healing long bones," J. Acoust. Soc. Am. 121, 3907-3921 (2007).

${ }^{19}$ L. De Marchi, A. Marzani, S. Caporale, and N. Speciale, "Ultrasonic guided-waves characterization with warped frequency transforms," IEEE Trans. Ultrason. Ferroelectr. Freq. Control 56, 2232-2240 (2009).

${ }^{20} \mathrm{~K}$. Xu, D. Ta and W. Wang, "Multiridge-based analysis for separating individual modes from multimodal guided wave signals in long bones," IEEE Trans. Ultrason. Ferroelectr. Freq. Control 57, 2480-2490 (2010).

${ }^{21}$ Z. Zhang, K. Xu, D. Ta, and W. Wang, "Joint spectrogram segmentation and ridge-extraction method for separating multimodal guided waves in long bones," Science China Physics, Mechanics and Astronomy 56(7), 1317-1323 (2013).

${ }^{22} \mathrm{~K}$. Xu, D. Ta, P. Moilanen, and W. Wang, "Mode separation of Lamb waves based on dispersion compensation method," J. Acoust. Soc. Am. 131, 2714-2722 (2012).

${ }^{23}$ Y. Yang, Z. K. Peng, W. M. Zhang, G. Meng, and Z. Q. Lang, "Dispersion analysis for broadband guided wave using generalized warblet transform," J. Sound Vib. 367, 22-36 (2016).

${ }^{24}$ L. Zeng, M. Zhao, J. Lin, and W. Wu, "Waveform separation and image fusion for Lamb waves inspection resolution improvement," NDT\&E Int. 79, 17-29 (2016).

${ }^{25}$ M. Ratassepp, A. Klauson, F. Chati, F. Léon, D. Décultot, G. Maze, and M. Fritzsche, "Application of orthogonality-relation for the separation of Lamb modes at a plate edge: Numerical and experimental predictions," Ultrasonics 57, 90-95 (2015).

${ }^{26}$ J. B. Harley and J. M. F. Moura, "Sparse recovery of the multimodal and dispersive characteristics of Lamb waves," J. Acoust. Soc. Am. 133, 2732 (2013).

${ }^{27}$ J. G. Minonzio, J. Foiret, M. Talmant, and P. Laugier, "Impact of attenuation on guided mode wavenumber measurement in axial transmission on bone mimicking plates," J. Acoust. Soc. Am. 130, 3574-3582 (2011).

${ }^{28}$ T. N. Tran, K. T. Nguyen, M. D. Sacchi, and L. H. Le, "Imaging ultrasonic dispersive guided wave energy in long bones 
using linear Radon transform," Ultrasound Med. Biol. 40, 2715-2727 (2014).

${ }^{29}$ T. N. Tran, L. H. Le, M. D. Sacchi, V. Nguyen, and E. H. Lou, "Multichannel filtering and reconstruction of ultrasonic guided wave fields using time intercept-slowness transform," J. Acoust. Soc. Am. 136, 248-259 (2014).

${ }^{30}$ C. H. Chapman, "Generalized Radon transforms and slant stacks," Geophys. J. Int. 66, 445-453 (1981).

${ }^{31}$ M. D. Sacchi, "Statistical and transform methods in geophysical signal processing," https://www.ualberta.ca/ msacchi/Notes.pdf, 209-253 (2002).

32 J. R. Thorson and J. F. Claerbout, "Velocity-stack and slant-stack stochastic inversion," Geophysics 50, 2727-2741 (1985).

${ }^{33}$ M. D. Sacchi and T. J. Ulrych, "High-resolution velocity gathers and offset space reconstruction," Geophysics 60, 1169 (1995).

${ }^{34}$ Y. Luo, J. Xia, R. D. Miller, Y. Xu, J. Liu, and Q. Liu, "Rayleigh-wave dispersive energy imaging using a high-resolution linear Radon transform," Pure Appl. Geophys. 165, 903-922 (2008).

${ }^{35}$ I. A. Viktorov, Rayleigh and Lamb waves: physical theory and applications, 67-75 (Plenum press New York, 1967).

36 Z. Su, L. Ye and Y. Lu, "Guided Lamb waves for identification of damage in composite structures: a review," J. Sound Vib. 295, 753-780 (2006).

${ }^{37}$ K. Xu, J. Minonzio, D. Ta, B. Hu, W. Wang, and P. Laugier, "Sparse SVD Method for High Resolution Extraction of the Dispersion Curves of Ultrasonic Guided Waves," IEEE Trans. Ultrason. Ferroelectr. Freq. Control, DOI: 10.1109/TUFFC.2016.2592688 (2016).

${ }^{38}$ L. Moreau, J. G. Minonzio, M. Talmant, and P. Laugier, "Measuring the wavenumber of guided modes in waveguides with linearly varying thickness," J. Acoust. Soc. Am. 135, 2614-2624 (2014).

${ }^{39}$ R. Schultz and Y. Jeffrey Gu, "Flexible, inversion-based Matlab implementation of the Radon transform," Comput. Geosci.-UK 52, 437-442 (2013).

${ }^{40}$ M. D. Sacchi, "Reweighting strategies in seismic deconvolution," Geophys. J. Int. 129, $651-656$ (1997).

${ }^{41}$ W. H. Press, S. A. Teukolsky, W. T. Vetterling, and B. P. Flannery, Numerical recipes 2nd edition: The art of scientific computing, 402-405 (Cambridge university press, 1992).

${ }^{42}$ H. W. Engl and W. Grever, "Using the L-curve for determining optimal regularization parameters," Numer. Math. 69, 25-31 (1994).

${ }^{43}$ F. Lefebvre, Y. Deblock, P. Campistron, D. Ahite, and J. J. Fabre, "Development of a new ultrasonic technique for bone and biomaterials in vitro characterization," J. Biomed. Mater. Res. 63, 441-446 (2002).

${ }^{44}$ D. Ta, K. Huang, W. Wang, Y. Wang, and L. H. Le, "Identification and analysis of multimode guided waves in tibia cortical bone," Ultrasonics 44, e279-e284 (2006).

45 P. Moilanen, P. Nicholson, V. Kilappa, S. Cheng, and J. Timonen, "Measuring guided waves in long bones: Modeling and experiments in free and immersed plates," Ultrasound Med. Biol. 32, 709-719 (2006).

46 J. J. Ditri and J. L. Rose, "Excitation of guided waves in generally anisotropic layers using finite sources," Journal of applied mechanics 61, 330-338 (1994).

${ }^{47}$ L. Wang, Y. Xu, J. Xia, and Y. Luo, "Effect of near-surface topography on high-frequency Rayleigh-wave propagation," J. Appl. Geophys. 116, 93-103 (2015).

${ }^{48}$ J. Xia, Y. Xu and R. D. Miller, "Generating an image of dispersive energy by frequency decomposition and slant stacking," Pure Appl. Geophys. 164, 941-956 (2007).

${ }^{49}$ F. Castanié, Spectral Analysis: parametric and non-parametric digital methods, 151-257 (John Wiley \& Sons, 2010).

${ }^{50}$ K. Xu, J. G. Minonzio, D. Ta, B. Hu, W. Wang, and P. Laugier, Sparse inversion SVD method for dispersion extraction of ultrasonic guided waves in cortical bone, in Corfu, 2015, p. 1-3.

${ }^{51}$ S. Yu, J. Ma, X. Zhang, and M. D. Sacchi, "Interpolation and denoising of high-dimensional seismic data by learning a tight frame," Geophysics 80, V119-V132 (2015).

${ }^{52}$ X. Song, D. Ta and W. Wang, "A base-sequence-modulated Golay code improves the excitation and measurement of ultrasonic guided waves in long bones," IEEE Trans. Ultrason. Ferroelectr. Freq. Control 59, 2580-2583 (2012).

53 J. Lin, J. Hua, L. Zeng, and Z. Luo, "Excitation Waveform Design for Lamb Wave Pulse Compression," IEEE Trans. Ultrason. Ferroelectr. Freq. Control 63, 165 - 177 (2015).

${ }^{54}$ M. Yucel, S. Fateri, M. Legg, A. Wilkinson, V. Kappatos, C. Selcuk, and T. Gan, "Coded waveform excitation for high resolution ultrasonic guided wave response," IEEE T. Ind. Inform., 1-1 (2016).

${ }^{55}$ K. Xu, D. Ta, B. Hu, P. Laugier, and W. Wang, "Wideband dispersion reversal of Lamb waves," IEEE Trans. Ultrason. Ferroelectr. Freq. Control 61, 997-1005 (2014).

${ }^{56}$ P. Moilanen, M. Talmant, V. Bousson, P. Nicholson, S. Cheng, J. Timonen, and P. Laugier, "Ultrasonically determined thickness of long cortical bones: Two-dimensional simulations of in vitro experiments," J. Acoust. Soc. Am. 122, 1818-1826 (2007).

${ }^{57}$ K. T. Nguyen, L. H. Le, T. N. Tran, M. D. Sacchi, and E. H. Lou, "Excitation of ultrasonic Lamb waves using a phased array system with two array probes: Phantom and in vitro bone studies," Ultrasonics 54, 1178-1185 (2014).

${ }^{58}$ E. Bossy, M. Talmant and P. Laugier, "Three-dimensional simulations of ultrasonic axial transmission velocity measurement on cortical bone models," J. Acoust. Soc. Am. 115, 2314-2324 (2004).

${ }^{59}$ J. Chen, J. Foiret, J. G. Minonzio, M. Talmant, Z. Su, L. Cheng, and P. Laugier, "Measurement of guided mode 
wavenumbers in soft tissue-bone mimicking phantoms using ultrasonic axial transmission," Phys. Med. Biol. 57, 3025-3037 (2012).

${ }^{60}$ J. G. Minonzio, J. Foiret, P. Moilanen, J. Pirhonen, Z. Zhao, M. Talmant, J. Timonen, and P. Laugier, "A free plate model can predict guided modes propagating in tubular bone-mimicking phantoms," J. Acoust. Soc. Am. 137, EL98-EL104 (2015).

${ }^{61}$ P. Moilanen, M. Talmant, V. Kilappa, P. Nicholson, S. L. Cheng, J. Timonen, and P. Laugier, "Modeling the impact of soft tissue on axial transmission measurements of ultrasonic guided waves in human radius," J. Acoust. Soc. Am. 124, 2364-2373 (2008).

${ }^{62}$ L. Cohen, "Time-frequency distributions-a review," P. IEEE 77, 941-981 (1989).

${ }^{63}$ J. Cardoso and A. Souloumiac, Blind beamforming for non-Gaussian signals, 1993 (IET), p. 362-370.

10

11 
List of tables:

Table I. Characteristics of ultrasonic signals in the long cortical bone at different $\boldsymbol{f} \cdot \boldsymbol{h}$ ranges

\begin{tabular}{|c|c|c|c|}
\hline \multirow{2}{*}{$\begin{array}{l}\text { frequency-thickness } \\
\text { product }(f \cdot h)(\mathrm{MHz} \\
\mathrm{mm})\end{array}$} & Case I & Case II & Case III \\
\hline & $f \cdot h<1$ & $1<f \cdot h<6$ & $6<f \cdot h$ \\
\hline Signal characteristics & $\begin{array}{l}\text { 1. Two fundamental } \\
\text { guided modes are } \\
\text { measured, i.e., a small } \\
\text { amplitude and fast } \\
\text { wave-packet } \\
\text { (symmetric S0) and a } \\
\text { high amplitude and } \\
\text { slow wave-packet } \\
\text { (asymmetric A0); } \\
\text { 2. Speed values are } \\
\text { different enough so that } \\
\text { the two wave-packets } \\
\text { do not overlap in time } \\
\text { even for relatively short } \\
\text { propagation distances } \\
\text { (a few cm). } \\
\text { The dispersion } \\
\text { information of the } \\
\text { lateral arrival A0 mode } \\
\text { can be used to estimate } \\
\text { cortical thickness }{ }^{11,56} \text {. }\end{array}$ & $\begin{array}{l}\text { 1. Under the wideband } \\
\text { excitation, more than } \\
5 \text { guided modes with } \\
\text { overlapping velocity } \\
\text { ranges; } \\
\text { 2. For the relatively } \\
\text { short propagation } \\
\text { distances (a few cm) } \\
8,12,18,44,57 \text {, a complete } \\
\text { dispersion extraction } \\
\text { of the overlapping } \\
\text { multimode is still } \\
\text { challenging. }\end{array}$ & 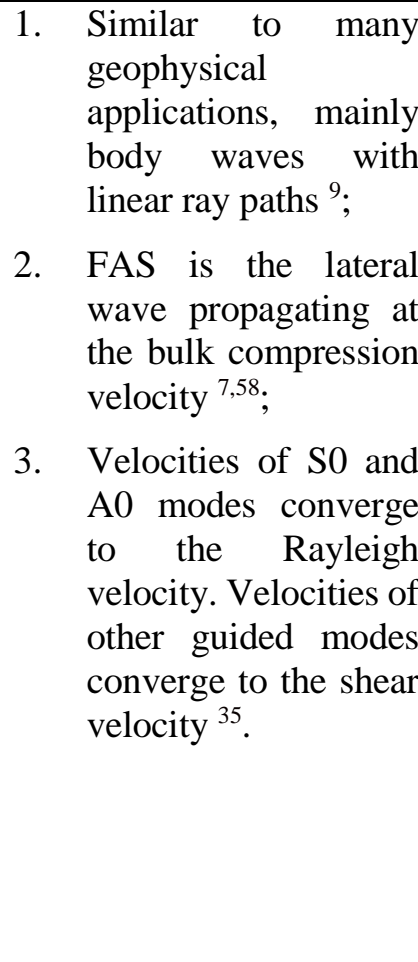 \\
\hline
\end{tabular}


Table II. Signal processing methods for assessment of the long cortical bone using axial transmission

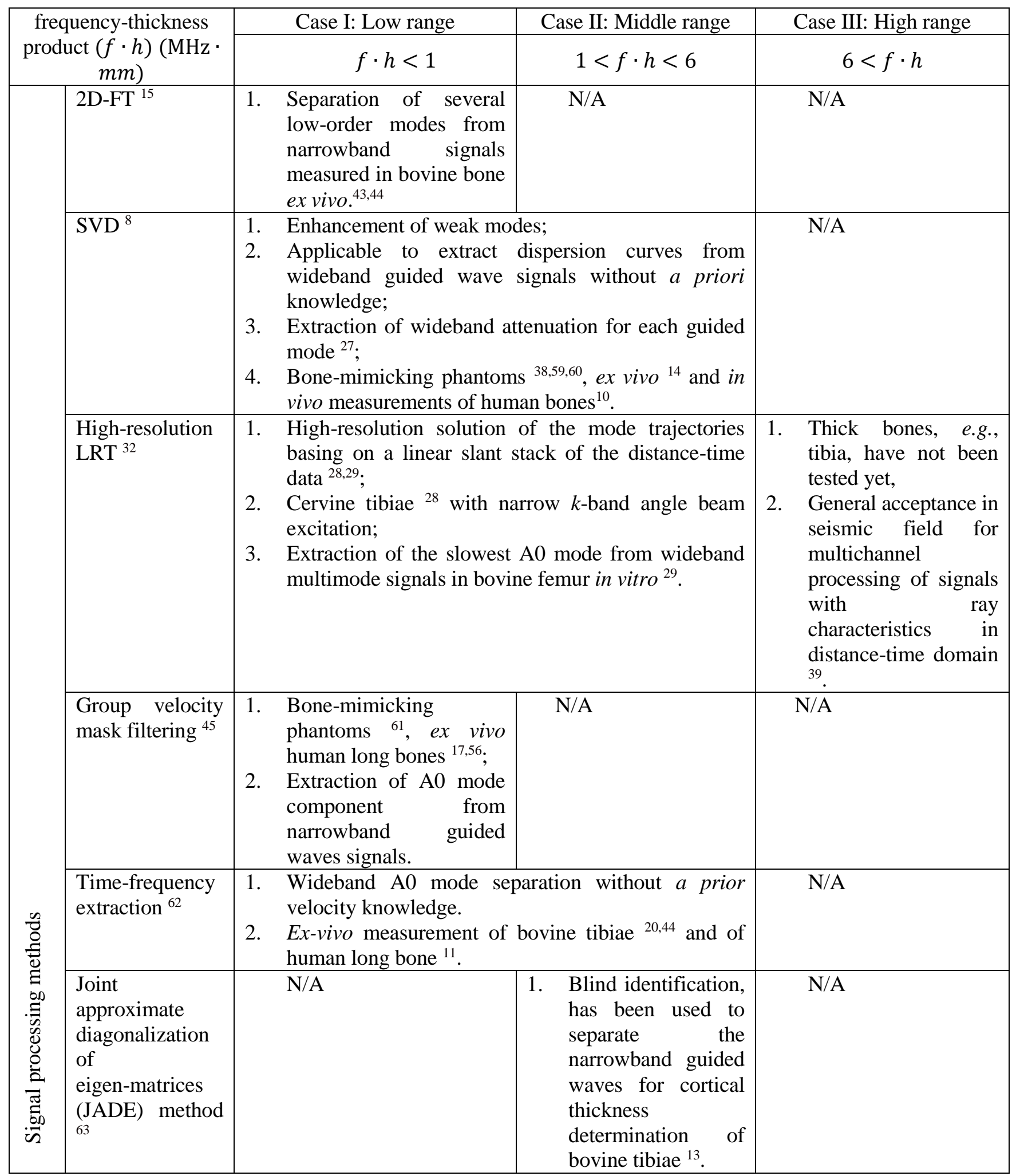

N/A: to the best knowledge of the authors, it has not been reported in the literatures of cortical bone guided waves processing. 
Table III. Shear and longitudinal velocities, density and thickness of the specimens used in experiments

\begin{tabular}{l|cccc}
\hline \multicolumn{1}{c|}{ Specimens } & $\rho\left(\mathrm{g} \cdot \mathrm{cm}^{-3}\right)$ & $h(\mathrm{~mm})$ & $V_{T}\left(\mathrm{~mm} \cdot \mu \mathrm{s}^{-1}\right)$ & $V_{L}\left(\mathrm{~mm} \cdot \mu \mathrm{s}^{-1}\right)$ \\
\hline Bone-mimicking plate $^{27}$ & 1.64 & 4 & 1.62 & $\left(V_{L \|}, V_{L \perp}\right)=(3.57,2.91)$ \\
PMMA $^{27}$ & 1.18 & 25.5 & 1.37 & 2.7 \\
Human radius specimen $^{14}$ & 1.85 & 1.58 & 1.8 & $\left(V_{L \|}, V_{L \perp}\right)=(4.0,3.41)$ \\
\hline
\end{tabular}


(a) Array Signal

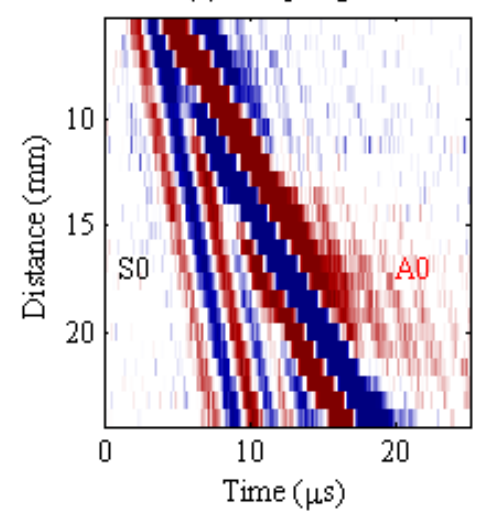

(d) $\tau-p\left(l_{2}\right)$

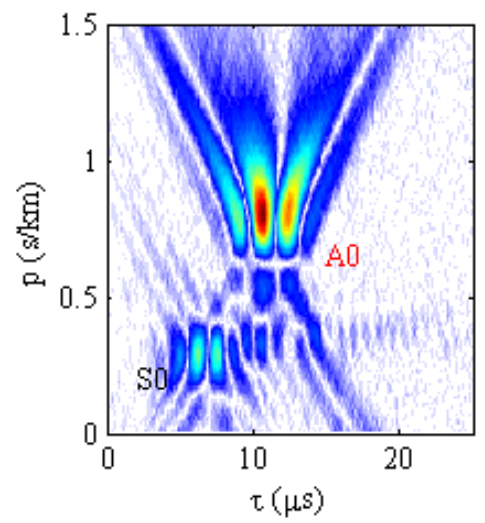

(b) $\mathrm{k}-\mathrm{f}(2 \mathrm{D}-\mathrm{FT})$

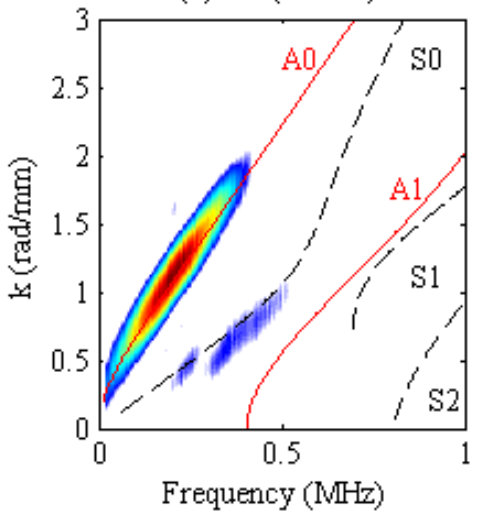

(e) $\tau-p$ return to $k-f\left(l_{1}\right)$

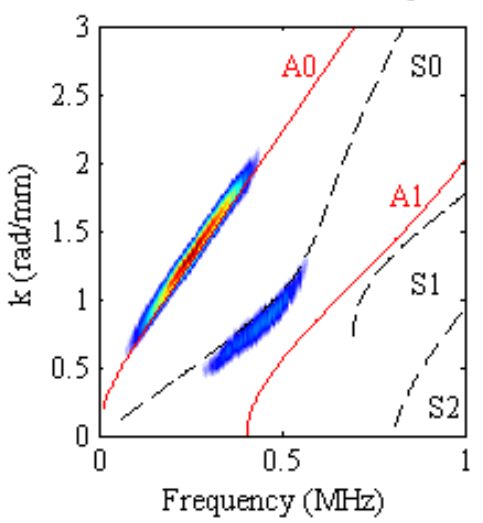

(c) k-f (SVD)
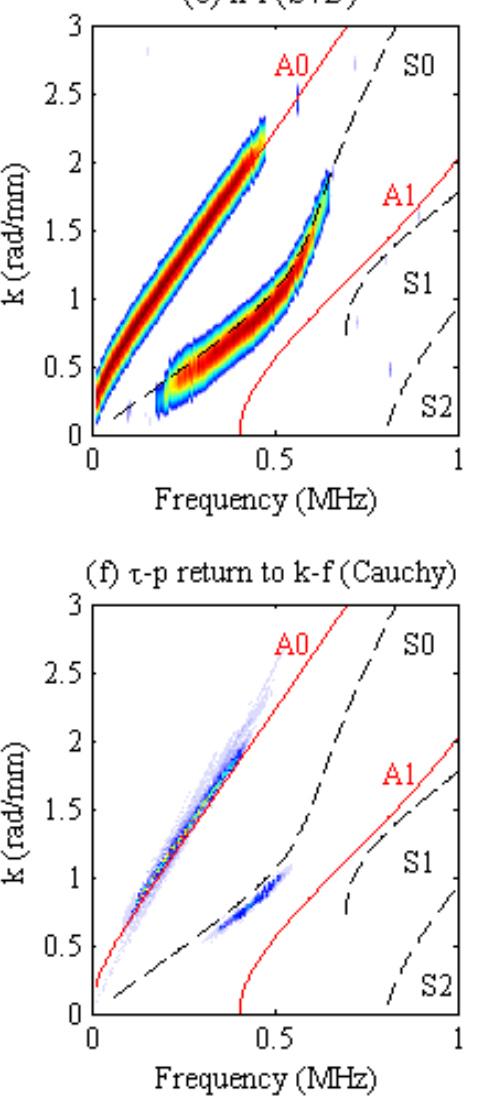

Fig. 1. (color online) Synthetic signals, narrow wavenumber-band Lamb modes A0 and S0 on a 2 mm-thick Sawbone plate with peak-to-peak amplitudes of 1 and 0.3, and SNR of 30dB, (a) distance-time diagram of the array-signals, (b) 2D-FT $k-f$ result, (c) SVD $k-f$ result, (d) the $\tau-p$ energy distributions obtained by LRT with $l_{2}$-norm, and $k-f$ results obtained by LRT with high-resolution regularization strategies, i.e., (e) $l_{1}$-norm, (f) Cauchy norm, respectively. 
(a) Array Signals

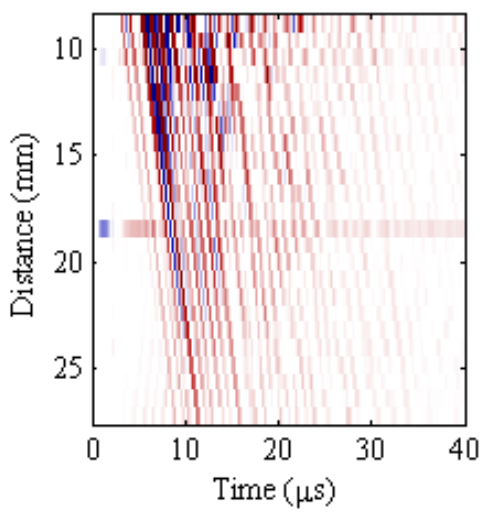

(d) $\tau-p\left(l_{2}\right)$

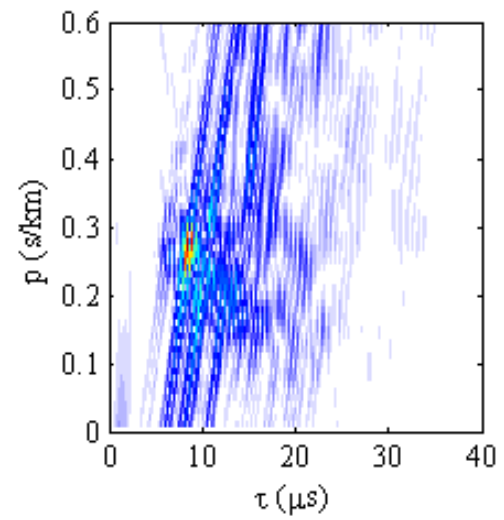

(b) $\mathrm{k}-\mathrm{f}(2 \mathrm{D}-\mathrm{FT})$

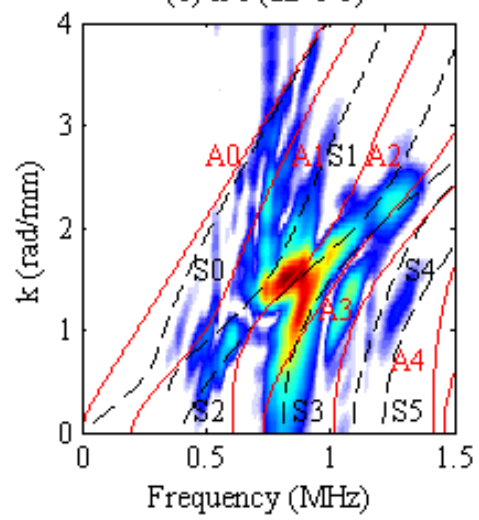

(e) $\tau-p$ return to $k-f\left(l_{l}\right)$

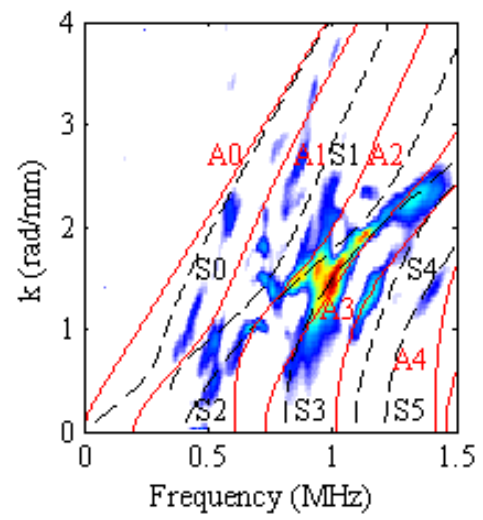

(c) k-f (SVD)
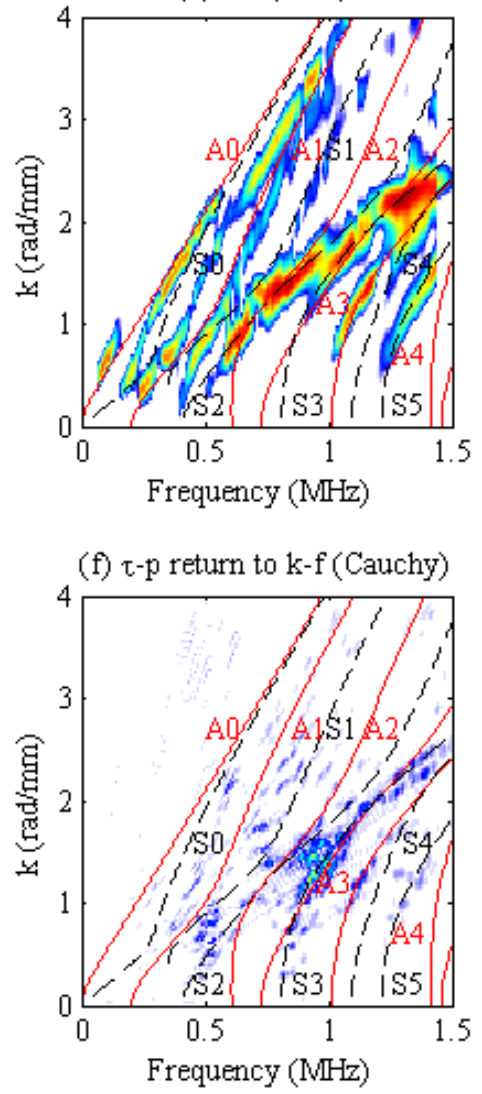

Fig. 2. (color online) Experimental signals measured in a $4 \mathrm{~mm}$-thick bone-mimicking plate, (a) distance-time diagram of the array-signals, (b) 2D-FT $k-f$ result, (c) SVD $k-f$ result, (d) the $\tau-p$ energy distributions obtained by LRT with $l_{2}$-norm, and $k-f$ results obtained by LRT with high resolution regularization strategies, i.e., (e) $l_{1}$-norm, (f) Cauchy norm, respectively. 
(a) Array Signals

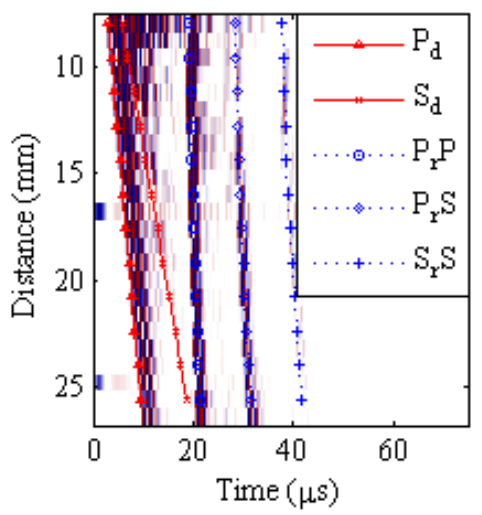

(d) $\tau-p\left(l_{2}\right)$

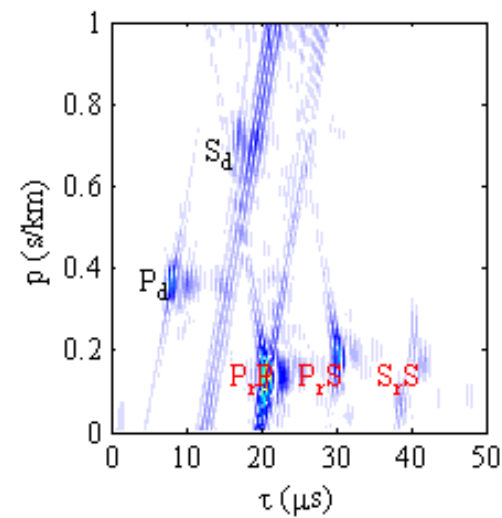

(b) $k-f(2 D-F T)$

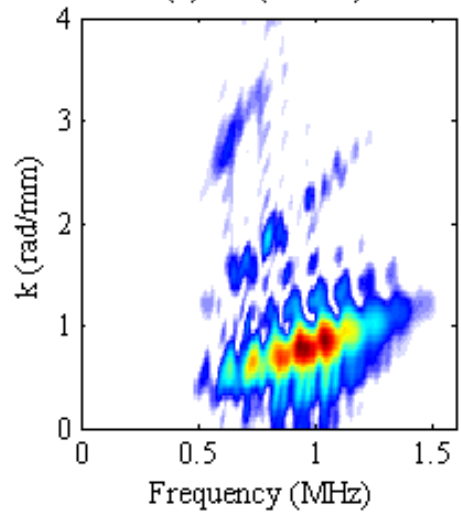

(e) $\tau-p$ return to $k-f\left(l_{l}\right)$

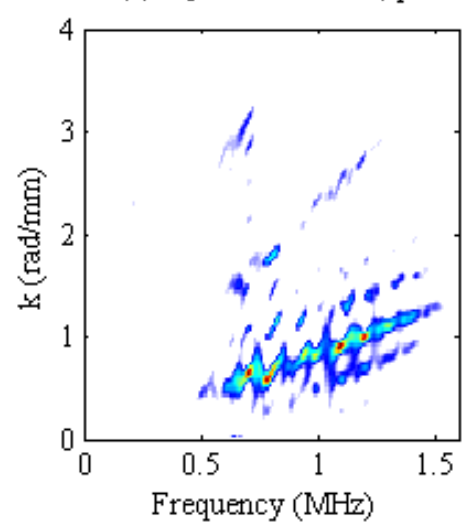

(c) $k-f(S V D)$
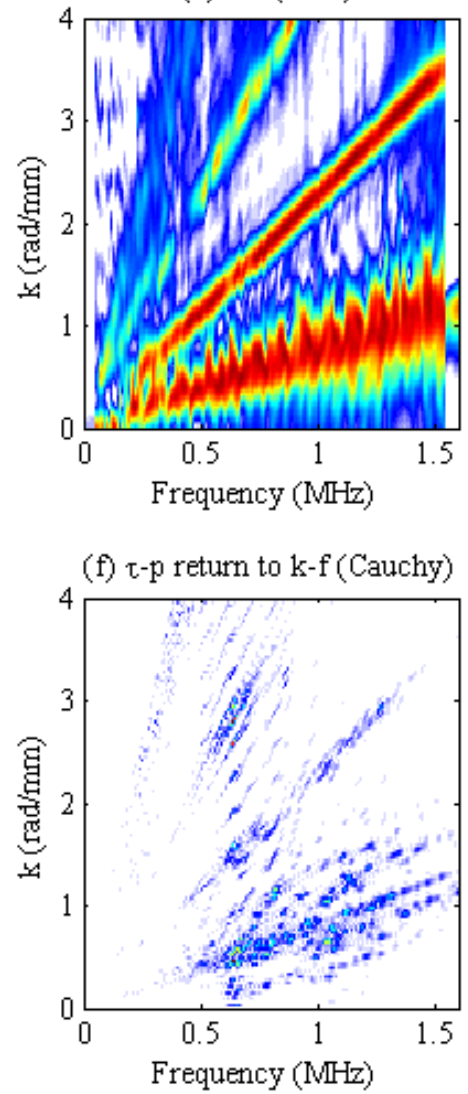

2

Fig. 3. (color online) Experimental signals measured in the $25.5 \mathrm{~mm}$-thick PMMA plate, (a) distance-time diagram of the synthetic array-signals, (b) 2D-FT $k$ - $f$ mode energy distribution, (c) SVD k-f mode energy distribution, (d) the $\tau-p$ energy distributions obtained by LRT with $l_{2}$-norm, and $k-f$ energy distributions obtained by LRT with high resolution regularization strategies, i.e., (e) $l_{1}$-norm, (f) Cauchy norm, respectively. 
(a) R-T Diagram (Cth $=2.5 \mathrm{~mm}$ )

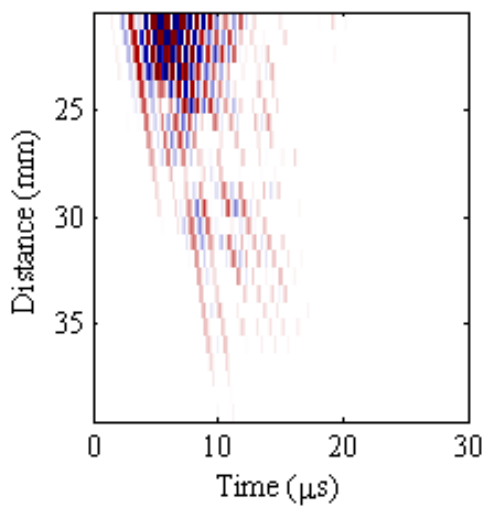

(d) $\tau-p\left(l_{2}\right)$

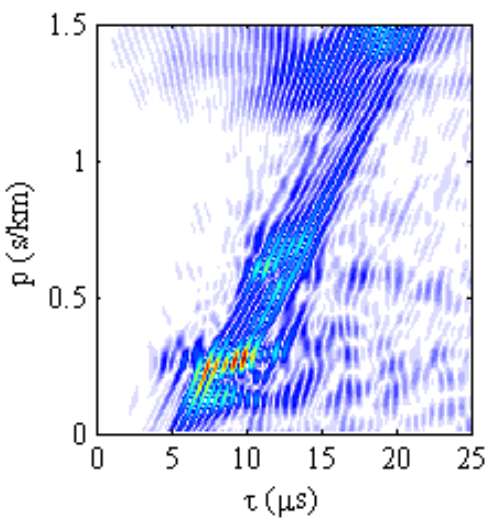

(b) $k-f(2 D-F T)$

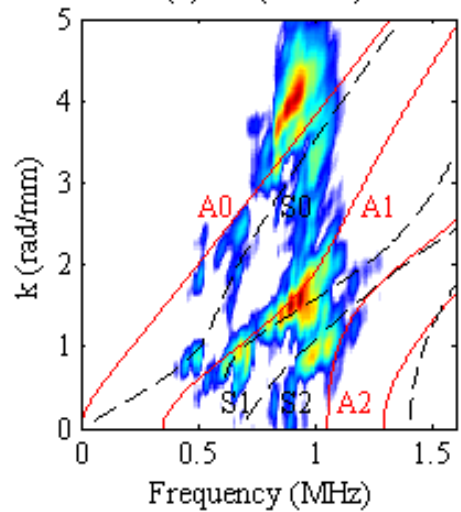

(e) $\tau$-p return to $k-f\left(l_{l}\right)$

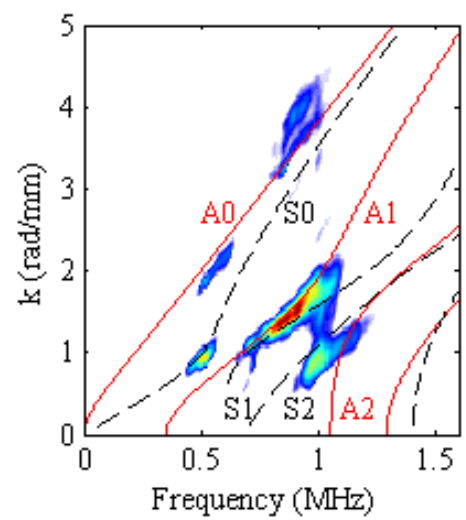

(c) $k$-f (SVD)
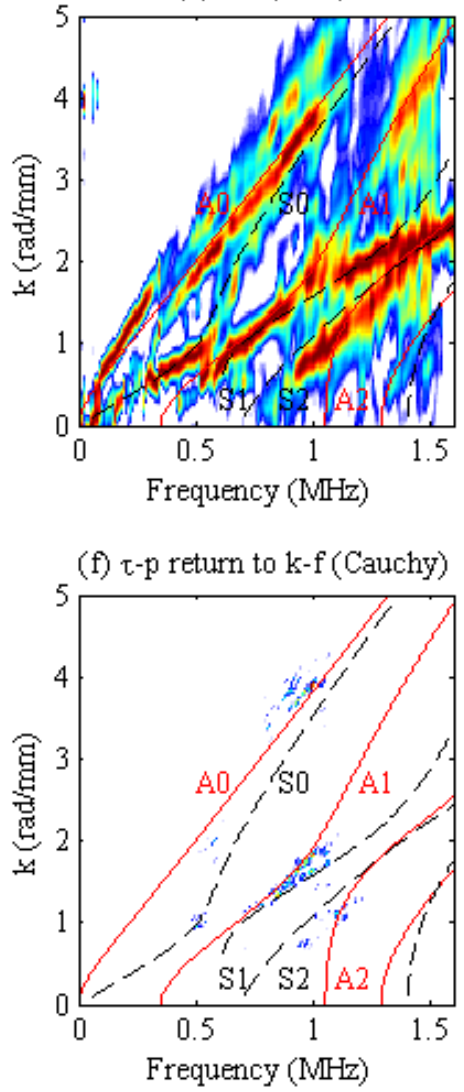

2

Fig. 4. (color online) Experimental guided signals measured in the $2.5 \mathrm{~mm}$-thick ex-vivo human radius, (a) distance-time diagram of the synthetic array-signals, (b) 2D-FT $k$ - $f$ mode energy distribution, (c) SVD $k-f$ mode energy distribution, (d) the $\tau-p$ energy distributions obtained by LRT with $l_{2}$-norm, and $k-f$ energy distributions obtained by LRT with high resolution regularization strategies, i.e., (e) $l_{1}$-norm, (f) Cauchy norm, respectively. 
(a) $l_{2}$ norm

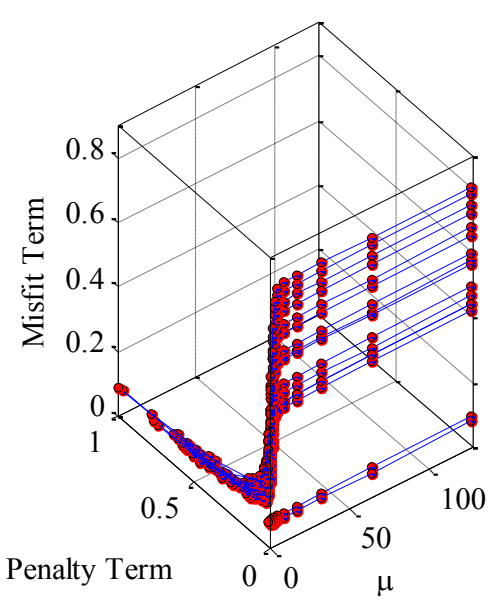

(b) $l_{l}$ norm

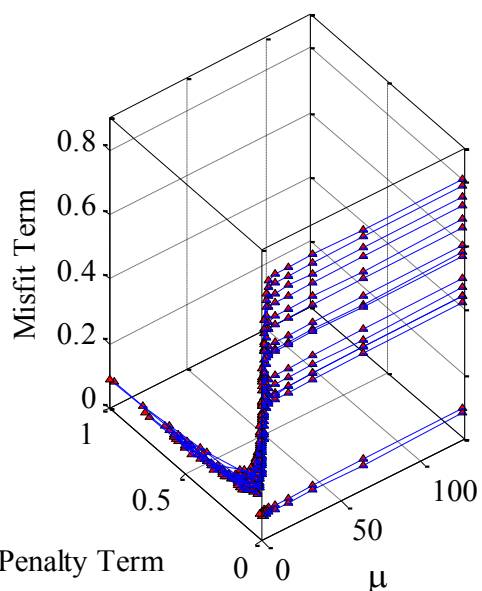

(c) Cauchy norm

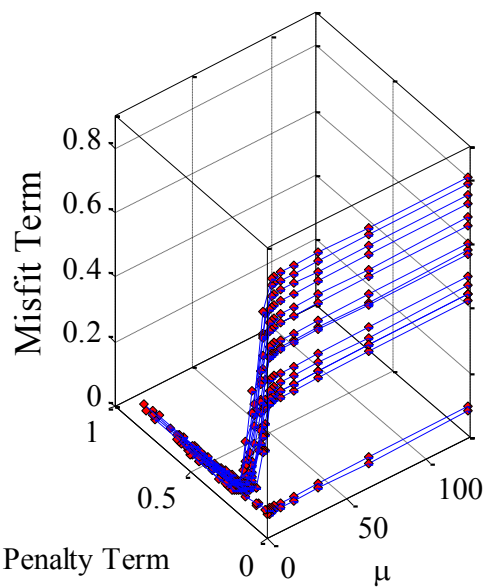

2

3

Fig. 5. (color online) L-curves of the synthetic signals (case I shown in Fig. 1), i.e., narrow wavenumber-band Lamb modes A0 and $\mathrm{S} 0$ on a 2 -mm-thick Sawbones plate, (a) $l_{2}$-norm, (b) $l_{1}$-norm, (c) Cauchy norm. The slowness $p$ and frequency f range from 0 to $2.56 \mu \mathrm{s} \cdot \mathrm{mm}^{-1}$ and 0.1 to $0.7 \mathrm{MHz}$, respectively. 
(a) $l_{2}$ norm

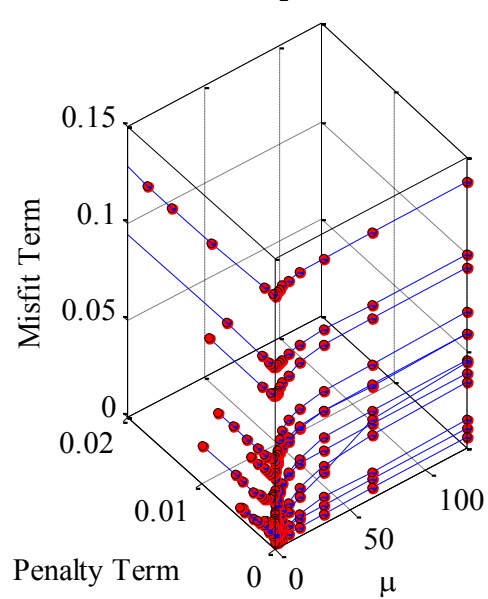

(b) $l_{l}$ norm

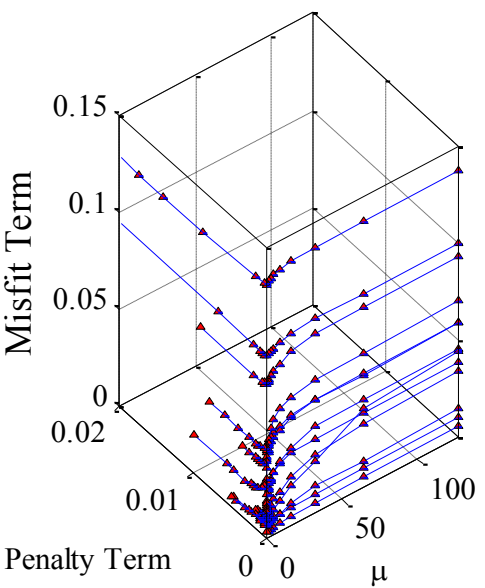

(c) Cauchy norm

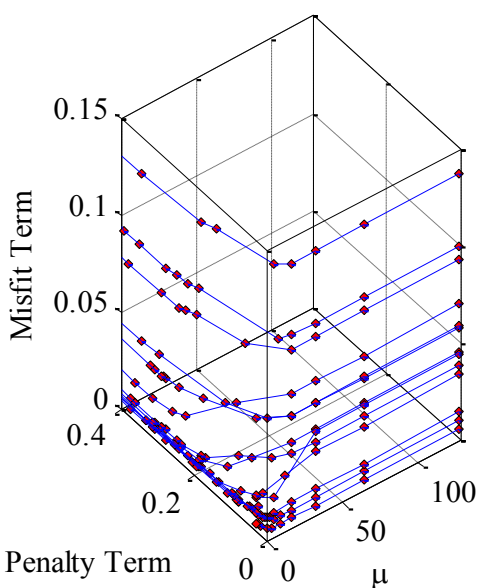

Fig. 6. (color online) L-curves of signals measured in a $2.5 \mathrm{~mm}$-thick human bone ex vivo (case II shown in Fig. 4), (a) $l_{2}$-norm, (b) $l_{1}$-norm, (c) Cauchy norm. The slowness $p$ and frequency $\mathrm{f}$ range from 0 to $2.56 \mu \mathrm{s} \cdot \mathrm{mm}^{-1}$ and 1.1 to $1.6 \mathrm{MHz}$, respectively. 
(a) Case I: Synthetic signals

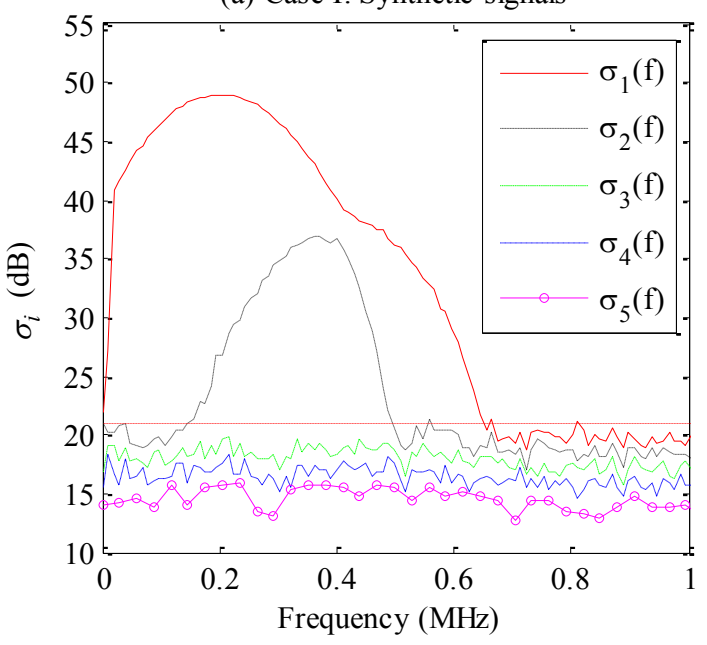

(b) Case II: 2.5 mm-thick ex-vivo bone

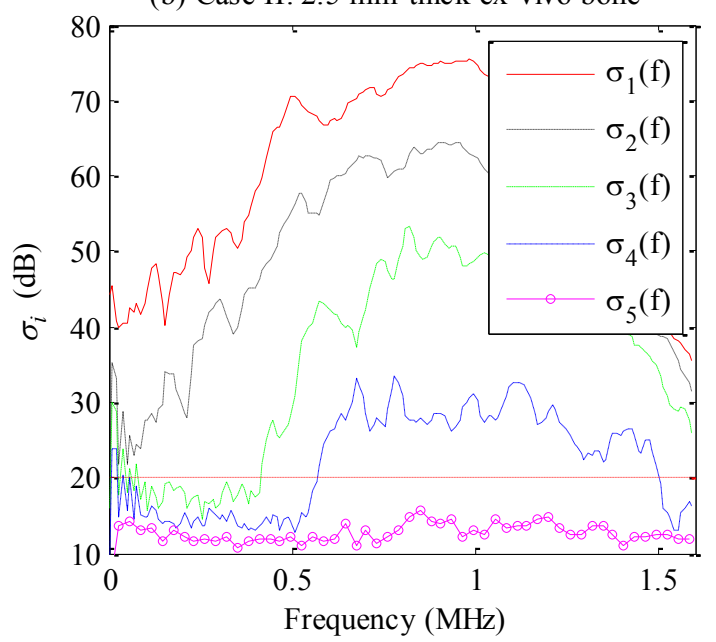

Fig. 7. (color online) Singular values $\sigma_{i}$ in dB scale versus frequency for (a) case I: synthetic signals, narrow wavenumber-band Lamb modes A0 and S0 on a 2-mm-thick Sawbones plate, (b) case II: 2.5 mm-thick human bone ex vivo. The five experimental singular values correspond to the number of emitters. The SNR threshold is shown with horizontal line. The singular values above the threshold define the signal subspace, and below the threshold the noise subspace. 